\title{
NUMERICAL METHODS FOR NASH EQUILIBRIA IN MULTIOBJECTIVE CONTROL OF PARTIAL DIFFERENTIAL EQUATIONS*
}

\author{
Angel Manuel Ramos \\ Departamento de Matemática Aplicada \\ Universidad Complutense de Madrid \\ Angel_Ramos@mat.ucm.es
}

\begin{abstract}
This paper is concerned with the numerical solution of multiobjective control problems associated with linear (resp., nonlinear) partial differential equations. More precisely, for such problems, we look for Nash equilibria, which are solutions to noncooperative games. First, we study the continuous case. Then, to compute the solution of the problem, we combine finite-difference methods for the time discretization, finiteelement methods for the space discretization, and conjugate gradient algorithms (resp., a suitable algorithm) for the iterative solution of the discrete control problems. Finally, we apply the above methodology to the solution of several tests problems.
\end{abstract}

Keywords: Partial differential equations, Heat equation, Burgers equation, optimal control, pointwise control, Nash equilibria, adjoint systems, conjugate gradient methods, multiobjective optimization, quasi-Newton algorithms.

\section{Introduction}

In this paper we present some methods for the numerical computation of the solutions of some multiobjective control problems associated with partial differential equations. The details about the results and algorithms showed here can be seen in [8], [9].

In a classical single-objective control problem for a system modelled by a Differential Equation, there is an output control $v$, acting on the

\footnotetext{
*Partial funding provided by the Spanish 'Plan Nacional de I+D+I (2000-2003) MCYT' through the AGL2000-1440-C02-01 project.
}

The original version of this chapter was revised: The copyright line was incorrect. This has been corrected. The Erratum to this chapter is available at DOI: 10.1007/978-0-387-35690-7_44 
equation and trying to achieve a pre-determined goal, usually consisting of minimizing a functional $J(\cdot)$.

In a multiobjective control problem there are more than one goal and, possibly, more than one control acting on the equation. Now, in contrast with the single-objective case, there are several strategies in order to choose the controls, depending of the character of the problem. These strategies can be cooperative (when the controls cooperate between them in order to achieve the goals), non-cooperative, hierarchical, etc..

Nash equilibria define a noncooperative multiple objective optimization strategy first proposed by Nash [6]. Since it originated in game theory and economics, the notion of player is often used. For an optimization problem with $G$ objectives (or functionals $J_{i}$ to minimize), a Nash strategy consists in having $G$ players (or controls $v_{i}$ ), each optimizing his own criterion. However, each player has to optimize his criterion given that all the other criteria are fixed by the rest of the players. When no player can further improve his criterion, it means that the system has reached a Nash Equilibrium state.

Of course there are other strategies for multiobjective optimization, such as the Pareto (cooperative) strategy [7] and the Stackelberg (hierarchical) strategy [10], etc..

Some previous works about these strategies for the control of partial differential equations are the following: In the articles by Lions [3]-[4] the author gives some results about the Pareto and Stackelberg strategies, respectively. In the article by Díaz and Lions [2], the authors prove an approximate controllability result for a system following a StackelbergNash strategy. In the article by Bristeau et al. [1], the authors compare Pareto and Nash strategies by using genetic algorithms to compute numerically the solutions corresponding to these strategies.

\section{Formulation of the problems}

\subsection{A linear case}

Let us consider $T>0$ and $\Omega \subset \mathbb{R}^{d}, d=1$ or 2 . We define $Q=$ $\Omega \times(0, T)$ and $\Sigma=\partial \Omega \times(0, T)$. We define the control spaces $\mathcal{U}_{1}=L^{2}\left(\omega_{1} \times\right.$ $(0, T))$ and $\mathcal{U}_{2}=L^{2}\left(\omega_{2} \times(0, T)\right)$, where $\omega_{1}, \omega_{2} \subset \Omega$ and $\omega_{1} \cap \omega_{2}=\emptyset$. Finally, we consider the functionals $J_{1}$ and $J_{2}$ given by

$$
\begin{aligned}
J_{i}\left(v_{1}, v_{2}\right)= & \frac{\alpha_{i}}{2}\left\|v_{i}\right\|_{\mathcal{U}}^{2}+\frac{k_{i}}{2}\left\|y\left(v_{1}, v_{2}\right)-y_{d, i}\right\|_{L^{2}\left(\omega_{d i} \times(0, T)\right)}^{2} \\
& +\frac{l_{i}}{2}\left\|y\left(v_{1}, v_{2} ; T\right)-y_{T, i}\right\|_{L^{2}\left(\omega_{T i}\right)}^{2}, \quad i=1,2
\end{aligned}
$$


for every $\left(v_{1}, v_{2}\right) \in \mathcal{U}_{1} \times \mathcal{U}_{2}$, where $\omega_{d i}, \omega_{T i} \subset \Omega(i=1,2)$ and function $y$ is defined as the solution of

$$
\begin{cases}\frac{\partial y}{\partial t}-\Delta y=f+v_{1} \chi_{\omega_{1}}+v_{2} \chi_{\omega_{2}} & \text { in } Q \\ y(x, 0)=y_{0}(x) & \text { in } \Omega \\ y=g & \text { on } \Sigma\end{cases}
$$

with $f, g, y_{0}, y_{d, i}$ and $y_{T, i}$ being smooth enough functions, $\alpha_{i}>0$, $k_{i}, l_{i} \geq 0$ and $k_{i}+l_{i}>0(i=1,2)$.

Remark 2.1 The following is also valid for more than two controls (and functionals), for more general linear operators, for different type of controls such as, for instance, boundary or initial controls and for different type of functionals.

Now, for every $w_{2} \in \mathcal{U}_{2}$ we consider the optimal control problem $\left(\mathcal{C} \mathcal{P}_{1}\left(w_{2}\right)\right):$ Find $u_{1}\left(w_{2}\right) \in \mathcal{U}_{1}$, such that

$$
J_{1}\left(u_{1}\left(w_{2}\right), w_{2}\right) \leq J_{1}\left(v_{1}, w_{2}\right), \forall v_{1} \in \mathcal{U}_{1} ;
$$

similarly for every $w_{1} \in \mathcal{U}_{1}$ we consider the optimal control problem $\left(\mathcal{C P}_{2}\left(w_{1}\right)\right)$ : Find $u_{2}\left(w_{1}\right) \in \mathcal{U}_{2}$, such that

$$
J_{2}\left(w_{1}, u_{2}\left(w_{1}\right)\right) \leq J_{2}\left(w_{1}, v_{2}\right), \forall v_{2} \in \mathcal{U}_{2} .
$$

The (unique) solution $u_{1}\left(w_{2}\right)$ (respectively $\left.u_{2}\left(w_{1}\right)\right)$ of $\left(\mathcal{C P}_{1}\left(w_{2}\right)\right)$ (respectively $\left.\left(\mathcal{C P}_{2}\left(w_{1}\right)\right)\right)$ is characterized by $\frac{\partial J_{1}}{\partial v_{1}}\left(u_{1}\left(w_{2}\right), w_{2}\right)=0$ (respectively $\left.\frac{\partial J_{2}}{\partial v_{2}}\left(w_{1}, u_{2}\left(w_{1}\right)\right)=0\right)$.

A Nash equilibrium is a pair $\left(u_{1}, u_{2}\right) \in \mathcal{U}_{1} \times \mathcal{U}_{2}$ such that $u_{1}=u_{1}\left(u_{2}\right)$ and $u_{2}=u_{2}\left(u_{1}\right)$, i.e. $\left(u_{1}, u_{2}\right)$ is a solution of the coupled system:

$$
\left\{\begin{array}{l}
\frac{\partial J_{1}}{\partial v_{1}}\left(u_{1}, u_{2}\right)=0 \\
\frac{\partial J_{2}}{\partial v_{2}}\left(u_{1}, u_{2}\right)=0
\end{array}\right.
$$

We show that system (3) has a unique solution. Furthermore, we give a numerical method for the solution of this problem and present the results obtained with this method on some examples.

Remark 2.2 A special case is when $\omega_{T 1} \cap \omega_{T 2} \neq \emptyset$ and/or $\omega_{d 1} \cap \omega_{d 2} \neq \emptyset$. This case is a competition-wise problem, with each control (or player) trying to reach (possibly) different goals over a common domain. In some sense this is the case where the behavior of the solution $y$ associated to the equilibrium $\left(u_{1}, u_{2}\right)$ is most difficult to forecast. 
It is obvious that the mapping

$$
\begin{aligned}
\left(\frac{\partial J_{1}}{\partial v_{1}}, \frac{\partial J_{2}}{\partial v_{2}}\right) & :\left(v_{1}, v_{2}\right) \in \mathcal{U}_{1} \times \mathcal{U}_{2} \longrightarrow \\
& \longrightarrow\left(\frac{\partial J_{1}}{\partial v_{1}}\left(v_{1}, v_{2}\right), \frac{\partial J_{2}}{\partial v_{2}}\left(v_{1}, v_{2}\right)\right) \in \mathcal{U}_{1} \times \mathcal{U}_{2}
\end{aligned}
$$

is an affine mapping of $V:=\mathcal{U}_{1} \times \mathcal{U}_{2}$. Therefore, there exist a linear continuous mapping $\mathcal{A} \in \mathcal{L}(V, V)$ and a vector $b \in V$ such that

$$
\left(\frac{\partial J_{1}}{\partial v_{1}}\left(v_{1}, v_{2}\right), \frac{\partial J_{2}}{\partial v_{2}}\left(v_{1}, v_{2}\right)\right)=\mathcal{A}\left(v_{1}, v_{2}\right)-b
$$

Let us identify mapping $\mathcal{A}$ : For every $\left(v_{1}, v_{2}\right) \in V$, the linear part of the affine mapping in relation (4) is defined by

$$
\mathcal{A}\left(v_{1}, v_{2}\right)=\left(\alpha_{1} v_{1}+p_{1} \chi_{\omega_{1}}, \alpha_{2} v_{2}+p_{2} \chi_{\omega_{2}}\right),
$$

where $p_{i}, i=1,2$, is the solution of

$$
\begin{cases}-\frac{\partial p_{i}}{\partial t}-\Delta p_{i}=k_{i} y \chi_{\omega_{d i}} & \text { in } Q \\ p_{i}(x, T)=l_{i} y(T) \chi_{\omega_{T i}} & \text { in } \Omega \\ p_{i}=0 & \text { on } \Sigma\end{cases}
$$

and $y$ is the solution of (2) with $f \equiv 0, y_{0} \equiv 0$ and $g \equiv 0$.

Proposition 2.1 Mapping $\mathcal{A}$ is linear, continuous, symmetric and strongly positive.

Let us identify $b$ : The constant part of the affine mapping (4) is the function $b \in V$ defined by $b=\left(p_{1} \chi_{\omega_{1}}, p_{2} \chi_{\omega_{2}}\right)$, where $p_{i}, i=1,2$, is the solution of

$$
\begin{cases}-\frac{\partial p_{i}}{\partial t}-\Delta p_{i}=k_{i}\left(Y-y_{d, i}\right) \chi_{\omega_{d i}} & \text { in } Q \\ p_{i}(x, T)=l_{i}\left(Y(T)-y_{T, i}\right) \chi_{\omega_{T i}} & \text { in } \Omega \\ p_{i}=0 & \text { on } \Sigma\end{cases}
$$

and $Y$ is the solution of (2) with $v_{1}=0$ and $v_{2}=0$.

Now, if we define $a(\cdot, \cdot): V \times V \rightarrow \mathbb{R}$ by

$$
a(v, w)=(\mathcal{A}(v), w)_{V} \quad \forall v, w \in V,
$$

and $L: V \rightarrow \mathbb{R}$ by

$$
L(v)=(b, v)_{V}, \quad \forall v \in V
$$


Proposition 2.1 proves that mapping $a(\cdot, \cdot)$ is bilinear continuous, symmetric and $V$-elliptic; mapping $L$ is (obviously) linear and continuous. Thus, system (3) has a unique solution, which can be computed by the following conjugate gradient algorihtm:

Step 1. $\left(u_{1}^{0}, u_{2}^{0}\right)$ is given in $V$.

Step 2.a. $y^{0}$ is the solution of (2) with $v_{1}=u_{1}^{0}$ and $v_{2}=u_{2}^{0}$.

Step 2.b. For $i=1,2, \begin{cases}-\frac{\partial p_{i}^{0}}{\partial t}-\Delta p_{i}^{0}=k_{i}\left(y^{0}-y_{i, d}\right) \chi_{\omega_{d i}} & \text { in } Q, \\ p_{i}^{0}(x, T)=l_{i}\left(\left(y^{0}(T)-y_{i, T}\right) \chi_{\omega_{T i}}\right. & \text { in } \Omega, \\ p_{i}^{0}=0 & \text { on } \Sigma .\end{cases}$

Step 2.c. $\left(g_{1}^{0}, g_{2}^{0}\right)=\left(\alpha_{1} u_{1}^{0}+p_{1}^{0} \chi_{\omega_{1}}, \alpha_{2} u_{2}^{0}+p_{2}^{0} \chi_{\omega_{2}}\right) \in V$.

Step 3. $\left(w_{1}^{0}, w_{2}^{0}\right)=\left(g_{1}^{0}, g_{2}^{0}\right) \in V$.

For $k \geq 0$, assuming that $\left(u_{1}^{k}, u_{2}^{k}\right),\left(g_{1}^{k}, g_{2}^{k}\right),\left(w_{1}^{k}, w_{2}^{k}\right)$ are known, we compute $\left(u_{1}^{k+1}, u_{2}^{k+1}\right),\left(g_{1}^{k+1}, g_{2}^{k+1}\right)$ and (if necessary) $\left(w_{1}^{k+1}, w_{2}^{k+1}\right)$ as follows:

Step 4.a. $\bar{y}^{k}$ is the solution of (2) with $f \equiv 0, y_{0} \equiv 0, g \equiv 0, v_{1}=w_{1}^{k}$ and $v_{2}=w_{2}^{k}$.

Step 4.b. For $i=1,2, \begin{cases}-\frac{\partial \bar{p}_{i}^{k}}{\partial t}-\Delta \bar{p}_{i}^{k}=k_{i} \bar{y}^{k} \chi_{\omega_{d i}} & \text { in } Q, \\ \bar{p}_{i}^{k}(x, T)=l_{i} \bar{y}^{k}(T) \chi_{\omega_{T i}} & \text { in } \Omega, \\ \bar{p}_{i}^{k}=0 & \text { on } \Sigma .\end{cases}$

Step 4.c. $\left(\bar{g}_{1}^{k}, \bar{g}_{2}^{k}\right)=\left(\alpha_{1} w_{1}^{k}+\bar{p}_{i}^{k} \chi_{\omega_{1}}, \alpha_{2} w_{2}^{k}+\bar{p}_{i}^{k} \chi_{\omega_{2}}\right)$.

Step 4.d. $\rho_{k}=\frac{\left\|\left(g_{1}^{k}, g_{2}^{k}\right)\right\|_{V}^{2}}{\int_{\omega_{1} \times(0, T)} \bar{g}_{1}^{k} w_{1}^{k} d x d t+\int_{\omega_{2} \times(0, T)} \bar{g}_{2}^{k} w_{2}^{k} d x d t}$.

Step 5. $\left(u_{1}^{k+1}, u_{2}^{k+1}\right)=\left(u_{1}^{k}, u_{2}^{k}\right)-\rho_{k}\left(w_{1}^{k}, w_{2}^{k}\right)$.

Step 6. $\left(g_{1}^{k+1}, g_{2}^{k+1}\right)=\left(g_{1}^{k}, g_{2}^{k}\right)-\rho_{k}\left(\bar{g}_{1}^{k}, \bar{g}_{2}^{k}\right)$.

If $\frac{\left\|\left(g_{1}^{k+1}, g_{2}^{k+1}\right)\right\|_{V}^{2}}{\left\|\left(g_{1}^{0}, g_{2}^{0}\right)\right\|_{V}^{2}} \leq \varepsilon$, then take $\left(u_{1}, u_{2}\right)=\left(u_{1}^{k+1}, u_{2}^{k+1}\right)$; else:

Step 7. $\gamma_{k}=\frac{\left\|\left(g_{1}^{k+1}, g_{2}^{k+1}\right)\right\|_{V}^{2}}{\left\|\left(g_{1}^{k}, g_{2}^{k}\right)\right\|_{V}^{2}}$.

Step 8. $\left(w_{1}^{k+1}, w_{2}^{k+1}\right)=\left(g_{1}^{k+1}, g_{2}^{k+1}\right)+\gamma_{k}\left(w_{1}^{k}, w_{2}^{k}\right)$.

Step 9. Do $k=k+1$, and go to Step 4.a.

\subsection{A nonlinear case}

We shall consider the Burgers equation with pointwise controls. All the results to follow are also valid for more than two control points but for simplicity we shall consider the case of only two control points $a_{1}$ and $a_{2}$. Let $Q=(0,1) \times(0, T)$. The state equation is 


$$
\begin{cases}y_{t}-\nu y_{x x}+y y_{x}=f+v_{1} \delta\left(x-a_{1}\right)+v_{2} \delta\left(x-a_{2}\right) & \text { in } Q \\ y_{x}(0, t)=0, y(1, t)=0 & \text { in }(0, T) \\ y(0)=y_{0} & \text { in }(0,1) .\end{cases}
$$

Let us consider $\omega_{d i}, \omega_{T i} \subset(0,1)(i=1,2)$ and the target functions $y_{d i} \in L^{2}\left(\omega_{d} \times(0, T)\right)$ and $y_{T i} \in L^{2}\left(\omega_{T}\right)(i=1,2)$. We take as the control space $\mathcal{U}_{1}=\mathcal{U}_{2}=\mathcal{U}=L^{2}(0, T)$.

The goal of each control $v_{i}(i=1,2)$ is to drive the solution $y$ close to $y_{d i}$ in $\omega_{d i} \times(0, T)$ and $y(T)$ close to $y_{T i}$ in $\omega_{T i}$ at a minimal cost for the control $v_{i}$. To do this, we define again two cost functions $J_{i}\left(v_{1}, v_{2}\right)$ as in (1).

For every $w_{1} \in \mathcal{U}_{1}$ and $w_{2} \in \mathcal{U}_{2}$ we consider the optimal control problems $\left(\mathcal{C P}_{1}\left(w_{2}\right)\right)$ and $\left(\mathcal{C P}_{2}\left(w_{1}\right)\right)$ as before. A Nash equilibrium is a pair $\left(u_{1}, u_{2}\right) \in \mathcal{U}_{1} \times \mathcal{U}_{2}$ such that $u_{1}=u_{1}\left(u_{2}\right)$ and $u_{2}=u_{2}\left(u_{1}\right)$.

The algorithm we propose is the following:

Step 1. $\left(u_{1}^{0}, u_{2}^{0}\right)$ is given in $\mathcal{U}_{1} \times \mathcal{U}_{2}$.

Step 2. We get $u_{1}^{1}$ as the solution of $\left(\mathcal{C P}_{1}\left(u_{2}^{0}\right)\right)$.

Step 3. We get $u_{2}^{1}$ as the solution of $\left(\mathcal{C P}_{2}\left(u_{1}^{0}\right)\right)$.

Then, for $k \geq 1$, assuming that $\left(u_{1}^{k}, u_{2}^{k}\right) \in \mathcal{U}_{1} \times \mathcal{U}_{2}$ is known, we compute $\left(u_{1}^{k+1}, u_{2}^{k+1}\right)$ as follows:

Step 4. If $u_{2}^{k}=u_{2}^{k-1}$ then $u_{1}^{k+1}=u_{1}^{k}$; else get $u_{1}^{k+1}$ as the solution of $\left(\mathcal{C P}_{1}\left(u_{2}^{k}\right)\right)$.

Step 5. If $u_{1}^{k}=u_{1}^{k-1}$ then $u_{2}^{k+1}=u_{2}^{k}$; else get $u_{2}^{k+1}$ as the solution of $\left(\mathcal{C P}_{2}\left(u_{1}^{k}\right)\right)$.

Step 6. If $u_{1}^{k+1}=u_{1}^{k}$ and $u_{2}^{k+1}=u_{2}^{k}$ then take $\left(u_{1}, u_{2}\right)=\left(u_{1}^{k+1}, u_{2}^{k+1}\right)$; else do $k=k+1$ and go to Step 4 .

Most of the descent methods for the numerical solution of $\left(\mathcal{C P} i\left(u_{j}^{k}\right)\right)$ will require the solution of the corresponding gradient, which we can be easily determined by a suitable adjoint system as in the previous linear case.

The following Remark is valid for both linear and nonlinear cases.

Remark 2.3 If $y_{d, 1}=y_{d, 2}=y_{d}, y_{T, 1}=y_{T, 2}=y_{T}, \alpha_{1}=\alpha_{2}=\alpha$, $k_{1}=k_{2}=k$ and $l_{1}=l_{2}=l$, then the Nash Equilibria problem (3) is equivalent to the classical control problem $(\mathcal{C P})$ : Find $\left(u_{1}, u_{2}\right) \in \mathcal{U}_{1} \times \mathcal{U}_{2}$, such that

$$
J\left(u_{1}, u_{2}\right) \leq J\left(v_{1}, v_{2}\right), \forall\left(v_{1}, v_{2}\right) \in \mathcal{U}_{1} \times \mathcal{U}_{2},
$$

where

$J\left(v_{1}, v_{2}\right)=\frac{\alpha}{2}\|v\|_{\mathcal{U}}^{2}+\frac{k}{2}\left\|y-y_{d}\right\|_{L^{2}\left(\omega_{d} \times(0, T)\right)}^{2}+\frac{l}{2}\left\|y(T)-y_{T}\right\|_{L^{2}\left(\omega_{T}\right)}^{2}$. 


\section{Time discretizations}

For simplicity, we consider from now on the special competition-wise control problem (see Remark 2.2) given by the case where $k_{1}=k_{2}=k$, $l_{1}=l_{2}=l, \omega_{d 1}=\omega_{d 2}=\omega_{d}$ and $\omega_{T 1}=\omega_{T 2}=\omega_{T}$.

\subsection{Linear case}

We point out that, for the special case specified above, the mapping $\mathcal{A}$ defined in Section 2.1 is $\mathcal{A}\left(v_{1}, v_{2}\right)=\left(\alpha_{1} v_{1}+p \chi_{\omega_{1}}, \alpha_{2} v_{2}+p \chi_{\omega_{2}}\right)$, with $p=p_{1}=p_{2}$ (since $p_{1}$ and $p_{2}$ are solution of the same equation). Further, the functions $\bar{p}_{1}^{k}$ and $\bar{p}_{2}^{k}$ defined in the Step 4.b of the Conjugate Gradient algorithm are solution of the same equation and therefore $\bar{p}_{1}^{k}=\bar{p}_{2}^{k}=\bar{p}^{k}$.

We consider the time discretization step $\Delta t$, defined by $\Delta t=T / N$, where $N$ is a positive integer. Then, if we denote $n \Delta t$ by $t^{n}$, we have $0<t^{1}<t^{2}<\cdots<t^{N}=T$. For simplicity, we assume that $f, g, y_{d, 1}$ and $y_{d, 2}$ are continuous functions, at least with respect to the time variable (if not we can always use continuous approximations of these functions). Now, we approximate $\mathcal{U}_{i}$ by $\mathcal{U}_{i}^{\Delta t}=\left(L^{2}\left(\omega_{i}\right)\right)^{N}, i=1,2$. Then, for every $w_{2} \in \mathcal{U}_{2}^{\Delta t}$ we approximate problem $\left(\mathcal{C P}_{1}\left(w_{2}\right)\right)$ by the following minimization problem $\left(\mathcal{C P}_{1}\left(w_{2}\right)\right)^{\Delta t}$ : Find $u_{1}^{\Delta t}\left(w_{2}\right) \in \mathcal{U}_{1}^{\Delta t}$, such that

$$
J_{1}^{\Delta t}\left(u_{1}^{\Delta t}\left(w_{2}\right), w_{2}\right) \leq J_{1}^{\Delta t}\left(v_{1}, w_{2}\right), \forall v_{1} \in \mathcal{U}_{1}^{\Delta t},
$$

with

$$
\begin{aligned}
J_{1}^{\Delta t}\left(v_{1}, v_{2}\right) & =\alpha_{1} \frac{\Delta t}{2} \sum_{n=1}^{N} \int_{\omega_{1}}\left|v_{1}^{n}\right|^{2} d x \\
+k & \frac{\Delta t}{2} \sum_{n=1}^{N} \int_{\omega_{d}}\left|y^{n}-y_{d, 1}\left(t^{n}\right)\right|^{2} d x+\frac{l}{2} \int_{\omega_{T}}\left|y^{N}-y_{T, 1}\right|^{2} d x,
\end{aligned}
$$

where $\left\{y^{n}\right\}_{n=1}^{N}$ is defined by the solution of the following semi-discrete parabolic problem:

$$
y^{0}=y_{0}
$$

and for $n=1, \ldots, N$,

$$
\begin{cases}\frac{y^{n}-y^{n-1}}{\Delta t}-\Delta y^{n}=f\left(t^{n}\right)+v_{1}^{n} \chi_{\omega_{1}}+v_{2}^{n} \chi_{\omega_{2}} & \text { in } \Omega, \\ y^{n}=g\left(t^{n}\right) & \text { in } \partial \Omega .\end{cases}
$$

Similarly, for every $w_{1} \in \mathcal{U}_{1}^{\Delta t}$, we approximate problem $\left(\mathcal{C P}_{2}\left(w_{1}\right)\right)$ by a minimization problem $\left(\mathcal{C P}_{2}\left(w_{1}^{\prime}\right)\right)^{\Delta t}$. Now, it can be proved that

$$
\frac{\partial}{\partial v_{i}} J_{i}^{\Delta t}\left(v_{1}, v_{2}\right)=\left\{\alpha_{i} v_{i}^{n}+p_{i}^{n} \chi_{\omega_{i}}\right\}_{n=1}^{N}
$$


for $i=1,2$, where $p_{i}^{N+1}=l\left(y^{N}\left(v_{1}, v_{2}\right)-y_{T, i}\right) \chi_{\omega_{T}}$, and for $n=N, \ldots, 1$,

$$
\begin{cases}\frac{p_{i}^{n}-p_{i}^{n+1}}{\Delta t}-\Delta p_{i}^{n}=k\left(y^{n}\left(v_{1}, v_{2}\right)-y_{d, i}\left(t^{n}\right)\right) \chi_{\omega_{d}} & \text { in } \Omega \\ p_{i}^{n}=0 & \text { on } \partial \Omega\end{cases}
$$

\subsection{Nonlinear case}

We approximate $\mathcal{U}$ by $\mathcal{U}^{\Delta t}=\mathbb{R}^{N}$ and problem $\left(\mathcal{C} \mathcal{P}_{1}\left(w_{2}\right)\right)$ by the following finite-dimensional minimization problem $\left(\mathcal{C P}_{1}\left(w_{2}\right)\right)^{\Delta t}$ : Find $u_{1}^{\Delta t}\left(w_{2}\right)=\left\{u_{1}^{n}\right\}^{n=1 \cdots N} \in \mathcal{U}^{\Delta t}$, such that

$$
\begin{gathered}
J_{1}^{\Delta t}\left(u_{1}^{\Delta t}, w_{2}\right) \leq J_{1}^{\Delta t}\left(v_{1}, w_{2}\right), \forall v_{1}=\left\{v_{1}^{n}\right\}^{n=1 \cdots N} \in \mathcal{U}^{\Delta t}, \\
J_{1}^{\Delta t}\left(v_{1}, v_{2}\right)=\alpha_{1} \frac{\Delta t}{2} \sum_{n=1}^{N}\left|v_{1}^{n}\right|^{2}+\frac{k \Delta t}{2} \sum_{n=1}^{N}\left\|y^{n}-y_{d, 1}(n \Delta t)\right\|_{L^{2}\left(\omega_{d 1}\right)}^{2} \\
+\frac{l}{2}\left((1-\theta)\left\|y^{N-1}-y_{T, 1}\right\|_{L^{2}\left(\omega_{T 1}\right)}^{2}+\theta\left\|y^{N}-y_{T, 1}\right\|_{L^{2}\left(\omega_{T 1}\right)}^{2}\right)
\end{gathered}
$$

where $\theta \in(0,1]$ and $\left\{y^{n}\right\}_{n=1}^{N}$ is defined from the solution of the following second order accurate time discretization scheme of (5):

$$
\begin{aligned}
& y^{0}=y_{0},
\end{aligned}
$$

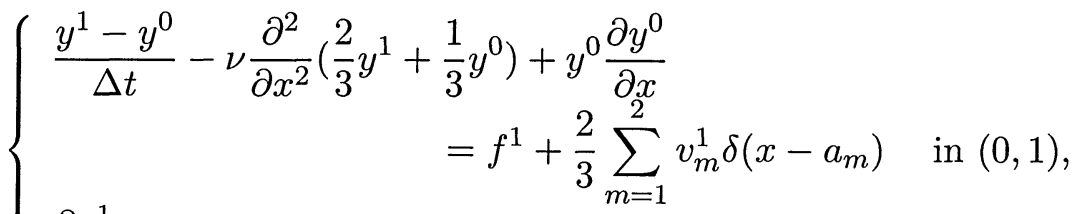

$$
\begin{aligned}
& \frac{\partial y^{1}}{\partial x}(0)=0, \quad y^{1}(1)=0,
\end{aligned}
$$

and for $n \geq 2$,

$$
\left\{\begin{array}{c}
\frac{\frac{3}{2} y^{n}-2 y^{n-1}+\frac{1}{2} y^{n-2}}{\Delta t}-\nu \frac{\partial^{2}}{\partial x^{2}} y^{n}+\left(2 y^{n-1}-y^{n-2}\right) \frac{\partial}{\partial x}\left(2 y^{n-1}-y^{n-2}\right) \\
=f^{n}+\sum_{m=1}^{2} v_{m}^{n} \delta\left(x-a_{m}\right) \text { in }(0,1), \\
\frac{\partial y^{n}}{\partial x}(0)=0, \quad y^{n}(1)=0 .
\end{array}\right.
$$

Similarly, we approximate $\left(\mathcal{C P}_{2}\left(w_{1}\right)\right)$ by $\left(\mathcal{C P}_{2}\left(w_{1}\right)\right)^{\Delta t}$. Again, the corresponding gradients can be computed by suitable adjoint systems.

\section{Numerical experiments}

In order to carry out numerical experiments we fully discretize the problems by adding a Finite Element Method to the time discretizations. 


\subsection{Linear case}

We consider $\Omega=(0,1) \times(0,1), \omega_{1}=(0,0.25) \times(0,0.25), \omega_{2}=$ $(0.75,1) \times(0,0.25), \omega_{T}=\Omega$ and $\omega_{d}=(0.25,0.75) \times(0.25,0.75)$ (see Figure 1). The space discretization step $h$ is defined by $h=1 /(I-1)$, where $I$ is a positive integer. Then, for every $i, j \in\{1, \cdots I\}$, we take the triangulation $\mathcal{T}_{h}$ with vertex $x_{i, j}=((i-1) h,(j-1) h)$ and the triangles as in the typical case showed in Figure 2.

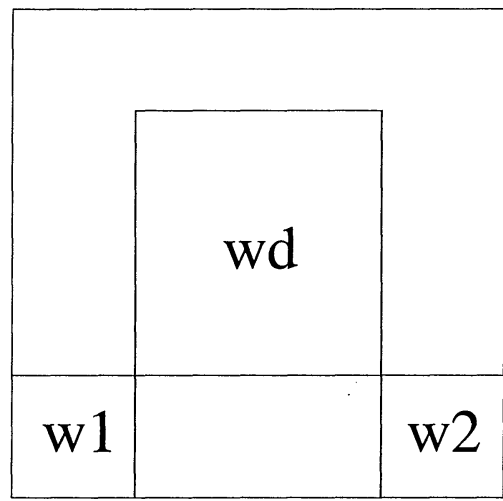

Figure 1. Control and observability domains of the problem.

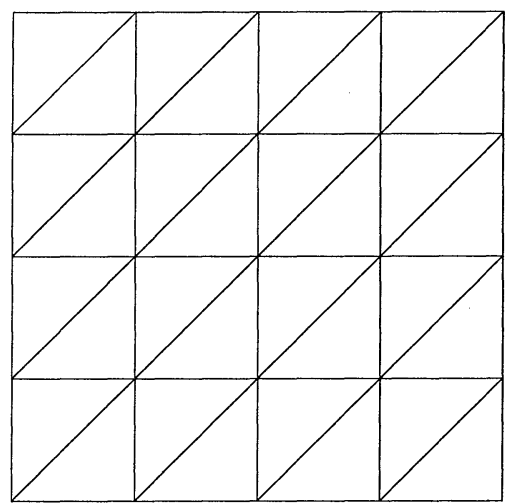

Figure 2. Typical finite element triangulation of $\Omega$.

For the data of the problem we take $f \equiv 1, y_{0} \equiv 0$ and $g=0$. In the conjugate gradient algorithm we take the initial guess $\left(u_{1}^{0}, u_{2}^{0}\right)=(0,0)$ and the stopping criterion $\varepsilon=10^{-8}$.

We consider the Stabilization Type Test Problem $k=1, l=0$ with finite horizon time $T=1.5, \Delta t=1.5 / 45$ and $h=1 / 36$. In order to see how the non-controlled solution behaves, we have visualized in Figure 3 the computed solution of the non-controlled equation at time $t=1.5$.

We consider the case of Different Goals: $y_{d, 1}=1, y_{d, 2}=-1$. In Figure 4 we have visualized the graph of the computed solution of the controlled equation with $\alpha_{1}=\alpha_{2}=10^{-6}$.

In Figures $5-6$ we have visualized the graph of $\|y(t)-1\|_{L^{2}\left(\omega_{d}\right)}^{2}$ and $\|y(t)-(-1)\|_{L^{2}\left(\omega_{d}\right)}^{2}$ for different cases. In Table 1 we give some further results about our solution.

Remark 4.1 We point out (see Figures 5-6 and Table 1) that, when the goals are different, the controlled solution can be worse, with respect to both goals, than the uncontrolled solution. 


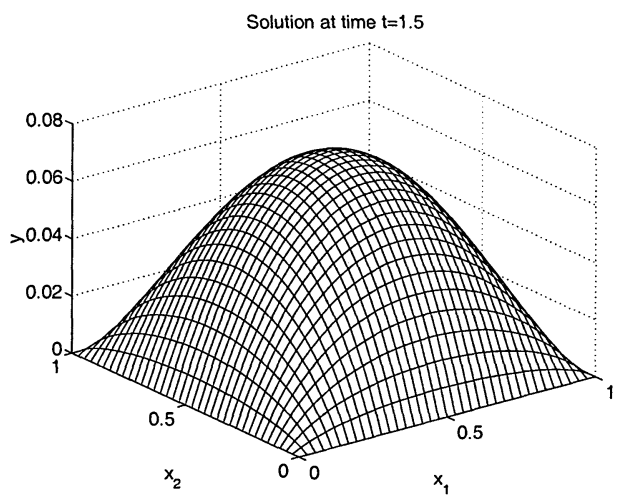

Figure 3. Noncontrolled solution at time $\mathrm{t}=1.5$.

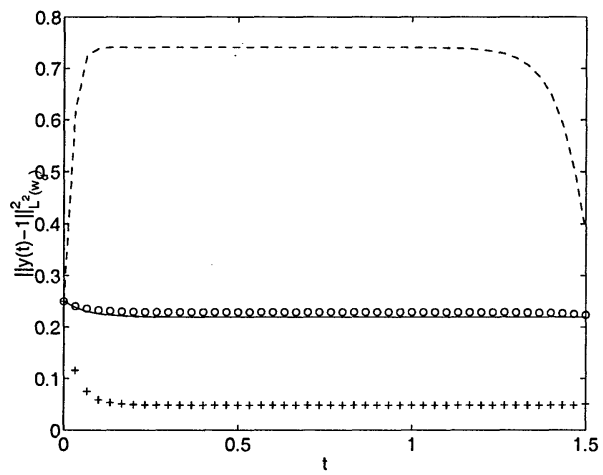

Figure 5. $\quad\|y(t)-1\|_{L^{2}\left(\omega_{d}\right)}^{2}, y$ is the computed solution for the following cases: uncontrolled equation $(-)$, $\alpha_{1}=\alpha_{2}=10^{-4}$ (oo), $\alpha_{1}=\alpha_{2}=10^{-6}$ $(--), \alpha_{1}=10^{-8}$ and $\alpha_{2}=10^{-2}(++)$.

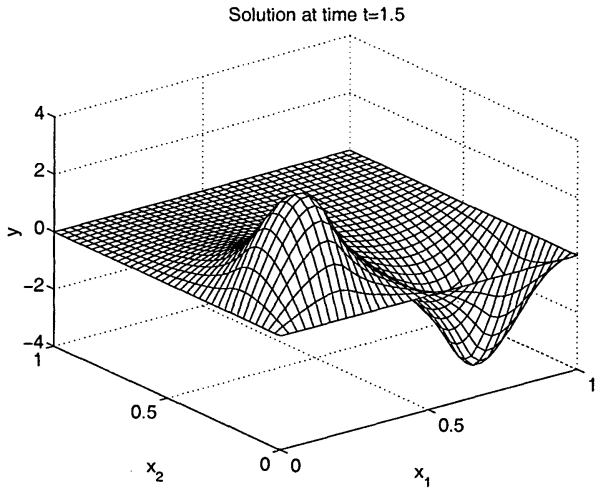

Figure 4. Computed solution of the controlled equation with $\alpha_{1}=$ $\alpha_{2}=10^{-6}$ at time $t=1.5$.

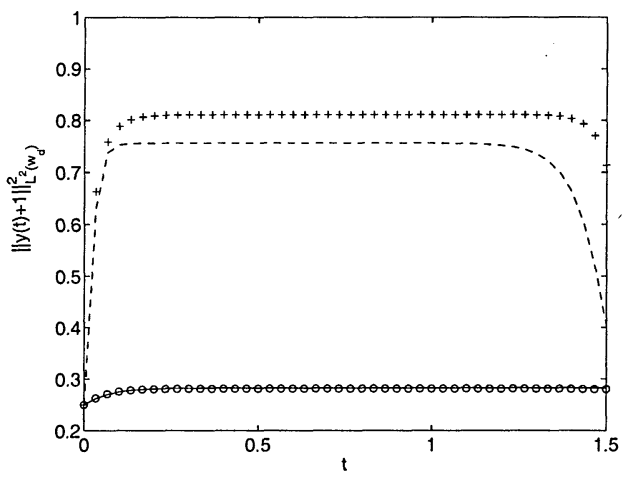

Figure 6. $\quad\|y(t)+1\|_{L^{2}\left(\omega_{d}\right)}^{2}, y$ is the computed solution for the following cases: uncontrolled equation $(-)$, $\alpha_{1}=\alpha_{2}=10^{-4}(\mathrm{oo}), \alpha_{1}=\alpha_{2}=10^{-6}$ $(--), \alpha_{1}=10^{-8}$ and $\alpha_{2}=10^{-2}(++)$.

\subsection{Nonlinear case}

We consider $T=1, a_{1}=1 / 5, a_{2}=3 / 5, I=128, N=256, \nu=10^{-2}$,

$$
f(x, t)= \begin{cases}1 & \text { if }(x, t) \in(0,1 / 2) \times(0, T), \\ 2(1-x) & \text { if }(x, t) \in[1 / 2,1) \times(0, T),\end{cases}
$$

$y_{0} \equiv 0$ and $\theta=3 / 2$. On each minimization problem of the algorithm, we get the sequence $u^{k}(k=1,2, \cdots)$ by using a quasi-Newton algorithm 
Table 1. Computational results for $y_{d, 1}=1, y_{d, 2}=-1$.

\begin{tabular}{|c||c|l|l|l|}
\hline & $\begin{array}{c}\text { No } \\
\text { control }\end{array}$ & $\begin{array}{l}\alpha_{1}=10^{-4} \\
\alpha_{2}=10^{-4}\end{array}$ & $\begin{array}{c}\alpha_{1}=10^{-8} \\
\alpha_{2}=10^{-2}\end{array}$ & $\begin{array}{l}\alpha_{1}=10^{-6} \\
\alpha_{2}=10^{-6}\end{array}$ \\
\hline \hline$\|y(t)-1\|_{L^{2}\left(\omega_{d} \times(0,1.5)\right)}^{2}$ & 0.330592 & 0.343811 & 0.0763473 & 1.07423 \\
\hline$\|y(t)+1\|_{L^{2}\left(\omega_{d} \times(0,1.5)\right)}^{2}$ & 0.422275 & 0.420292 & 1.20273 & 1.09692 \\
\hline
\end{tabular}

à la BFGS (see [5]). We stop iterating after step $k$ if either

$$
\begin{gathered}
\left\|\frac{\partial J_{h}^{\Delta t}}{\partial v}\left(u^{k}\right)\right\|_{\infty} \leq 10^{-5}, \quad \text { or } \\
\frac{J_{h}^{\Delta t}\left(u^{k-1}\right)-J_{h}^{\Delta t}\left(u^{k}\right)}{\max \left\{\left|J_{h}^{\Delta t}\left(u^{k-1}\right)\right|,\left|J_{h}^{\Delta t}\left(u^{k}\right)\right|, 1\right\}} \leq 2 \cdot 10^{-9} .
\end{gathered}
$$

We consider the Controllability Type Test Problem $\alpha_{1}=\alpha_{2}=1, k=0$, $l=8$. For the case $y_{T 1}(x)=\frac{1}{2}\left(1-x^{3}\right), y_{T 2}(x)=1-x^{3}$, Figure 7 shows the uncontrolled state solution $y(T)(\ldots)$, the target functions $y_{T 1}(---)$, $y_{T 2}(-.-)$, and the controlled state solution $y(T)(-)$, when controlling with a Nash strategy. Figure 8 shows the computed controls. In Table 2 we give some further information about several tests.

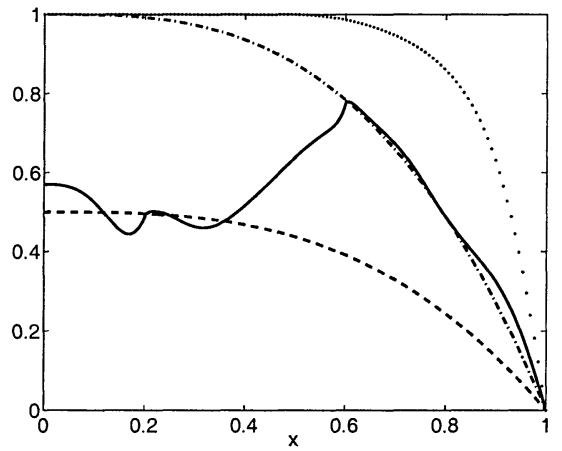

Figure 7. The target functions $y_{T 1}$ (- -), $y_{T 2}(-$. -), the uncontrolled (..) and controlled (-) states, for the Nash strategy, at time $T$.

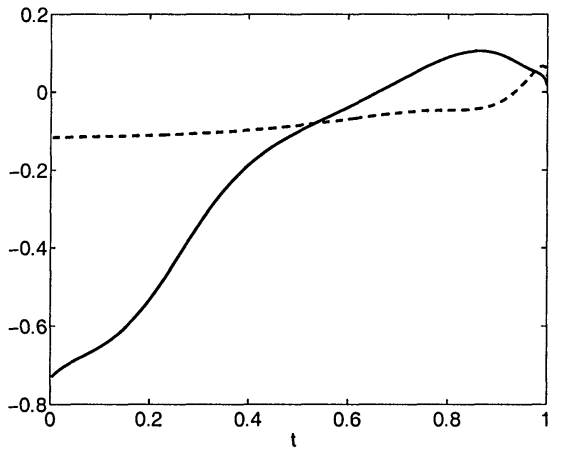

Figure 8. The computed controls $u_{1}(-)$ and $u_{2}(--)$ for the Nash strategy.

\section{Acknowledgments}

The author wishes to thank Prof. R. Glowinski and Dr. J. Periaux for their encouragement and support. 
Table 2. Computational results for the Nash strategy. NQNM= Number of times the Quasi-Newton Method has been used for each functional. NPES= Number of parabolic equations solved for each functional. Test 1: $y_{T 1}(x)=y_{T 2}(x)=1-x^{3}$. Test 2: $y_{T 1}(x)=\frac{1}{2}\left(1-x^{3}\right)$ and $y_{T 2}(x)=1-x^{3}$. Test 3: $y_{T 1}(x)=1-x^{3}$ and $y_{T 2}(x)=\frac{9}{8}\left(1-x^{6}\right)$. Test $4: y_{T 1}(x)=\frac{9}{8}\left(1-x^{6}\right)$ and $y_{T 2}(x)=1-x^{3}$.

\begin{tabular}{|c|c|c|c|c|}
\hline & Test 1 & Test 2 & Test 3 & Test4 \\
\hline "NQNM $J_{1} / J_{2}$ & $19 / 18$ & $5 / 4$ & $6 / 6$ & $55 / 55$ \\
\hline NPES $J_{1} / J_{2}$ & 286 / 232 & $188 / 54$ & $78 / 76$ & $1576 / 700$ \\
\hline$\frac{\left\|y(0 ; T)-y_{T 1}\right\|}{\left\|y_{T 1}\right\|}$ & 0.2522 & 1.3308 & 0.2522 & 0.1001 \\
\hline$\frac{\| y(u ; T)-y_{T 1}}{\left\|y_{T 1}\right\|}$ & 0.0241 & 0.4921 & 0.2288 & 0.1702 \\
\hline $\begin{array}{c}y(0 ; T)-y_{T 2} \\
\left\|y_{T 2}\right\| \\
\end{array}$ & 0.2522 & 0.2522 & 0.1001 & 0.2522 \\
\hline$\frac{y(u ; T)-y_{T 2}}{\left\|y_{T 2}\right\|}$ & 0.0241 & 0.4110 & 0.1445 & 0.2395 \\
\hline$u_{1}$ & 0.0540 & 0.3371 & 0.1334 & 1.1486 \\
\hline$u_{2}$ & 0.0944 & 0.0850 & 0.0849 & 0.9983 \\
\hline
\end{tabular}

\section{References}

[1] Bristeau, M.O., Glowinski, R., Mantel, B., Periaux, J., and Sefrioui, M., Genetic Algorithms for Electromagnetic Backscattering Multiobjective Optimization. In Electromagnetic Optimization by Genetic Algorithms, Edited by Y. RahmatSamii and E. Michielssen, John Wiley, New York, pp. 399-434, 1999.

[2] Díaz, J.I. and Lions, J.L., On the Approximate Controllability of StackelbergNash Strategies. In Mathematics and Environment, Lecture Notes, SpringerVerlag, 2001.

[3] Lions, J.L., Contrôle de Pareto de Systèmes Distribués: Le Cas d'Évolution, Comptes Rendus de l'Académie des Sciences, Serie I, 302, 413-417, 1986.

[4] Lions, J.L. Some Remarks on Stackelberg's Optimization, Mathematical Models and Methods in Applied Sciences, 4, 477-487, 1994.

[5] Liu, D.C., and Nocedal, J., On the Limited Memorey BFGS Method for LargeScale Optimization, Mathematical Programming, 45, 503-528, 1989.

[6] Nash, J.F., Noncooperative Games, Annals of Mathematics, 54, 286-295, 1951.

[7] Pareto, V., Cours d'Économie Politique, Rouge, Lausanne, Switzerland, 1896.

[8] Ramos, A.M., Glowinski, R., and Periaux, J., Nash Equilibria for the Multiobjective Control of Linear Partial Differential Equations, Journal of Optimization, Theory and Applications, 112, No. 3, 457-498, 2002.

[9] Ramos, A.M., Glowinski, R., and Periaux, J., Pointwise Control of the Burgers Equation and Related Nash Equilibrium Problems: Computational Approach, Journal of Optimization, Theory and Applications, 112, No. 3, 499-516, 2002.

[10] von Stackelberg, H., Marktform und Gleichgewicht, Springer, Berlin, Germany, 1934. 